\title{
Performance of neural network basecalling tools for Oxford Nanopore sequencing
}

\author{
Ryan R. Wick ${ }^{*}$ (1D, Louise M. Judd ${ }^{1}$ and Kathryn E. Holt ${ }^{1,2}$
}

\begin{abstract}
Background: Basecalling, the computational process of translating raw electrical signal to nucleotide sequence, is of critical importance to the sequencing platforms produced by Oxford Nanopore Technologies (ONT). Here, we examine the performance of different basecalling tools, looking at accuracy at the level of bases within individual reads and at majority-rule consensus basecalls in an assembly. We also investigate some additional aspects of basecalling: training using a taxon-specific dataset, using a larger neural network model and improving consensus basecalls in an assembly by additional signal-level analysis with Nanopolish.

Results: Training basecallers on taxon-specific data results in a significant boost in consensus accuracy, mostly due to the reduction of errors in methylation motifs. A larger neural network is able to improve both read and consensus accuracy, but at a cost to speed. Improving consensus sequences ('polishing') with Nanopolish somewhat negates the accuracy differences in basecallers, but pre-polish accuracy does have an effect on post-polish accuracy.

Conclusions: Basecalling accuracy has seen significant improvements over the last 2 years. The current version of ONT's Guppy basecaller performs well overall, with good accuracy and fast performance. If higher accuracy is required, users should consider producing a custom model using a larger neural network and/or training data from the same species.
\end{abstract}

Keywords: Oxford Nanopore, Basecalling, Long-read sequencing

\section{Background}

Oxford Nanopore Technologies (ONT) long-read sequencing is based on the following concept: pass a single strand of DNA through a membrane via a nanopore and apply a voltage difference across the membrane. The nucleotides present in the pore will affect the pore's electrical resistance, so current measurements over time can indicate the sequence of DNA bases passing through the pore. This electrical current signal (a.k.a. the 'squiggle' due to its appearance when plotted) is the raw data gathered by an ONT sequencer. Basecalling for ONT devices is the process of translating this raw signal into a DNA sequence. It is not a trivial task as the electrical signals come from single molecules, making for noisy and

*Correspondence: rrwick@gmail.com

'Department of Infectious Diseases, Central Clinical School, Monash University, Melbourne 3004, Australia

Full list of author information is available at the end of the article stochastic data. Furthermore, the electrical resistance of a pore is determined by the bases present within multiple nucleotides that reside in the pore's narrowest point (approximately five nucleotides for the R9.4 pore), yielding a large number of possible states: $4^{5}=1024$ for a standard four-base model. When modified bases are present, e.g. 5-methylcytosine, the number of possible states can grow even higher: $5^{5}=3125$. This makes basecalling of ONT device signals a challenging machine learning problem and a key factor determining the quality and usability of ONT sequencing.

Basecalling is an active field, with both ONT and independent researchers developing methods. Modern basecallers all use neural networks, and these networks must be trained using real data. The performance of any particular basecaller is therefore influenced by the data used to train its model. This is especially relevant when basecalling native (not PCR-amplified) DNA, which can contain

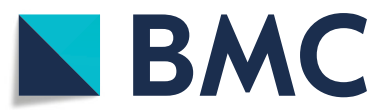

(c) The Author(s). 2019 Open Access This article is distributed under the terms of the Creative Commons Attribution 4.0 International License (http://creativecommons.org/licenses/by/4.0/), which permits unrestricted use, distribution, and reproduction in any medium, provided you give appropriate credit to the original author(s) and the source, provide a link to the Creative Commons license, and indicate if changes were made. The Creative Commons Public Domain Dedication waiver (http://creativecommons.org/publicdomain/zero/1.0/) applies to the data made available in this article, unless otherwise stated. 
base modifications. A basecaller's performance in such a case may depend on whether the modifications and their sequence motifs were represented in its training set.

Basecalling accuracy can be assessed at the read level (read accuracy) or in terms of accuracy of the consensus sequence (consensus accuracy). Read accuracy measures the sequence identity of individual basecalled reads relative to a trusted reference. Consensus accuracy measures the identity of a consensus sequence constructed from multiple overlapping reads originating from the same genomic location. Consensus accuracy generally improves with increased read depth, e.g. a consensus built from 10 reads is likely to be less accurate than one built from 100 reads.

While read and consensus accuracy may be correlated, this relationship is not guaranteed. I.e. more accurate reads do not necessarily produce a more accurate consensus. Random read errors are unlikely to appear in the consensus, as they occur in the minority of reads at their locus. Systematic errors that occur in many reads can however appear in the consensus. Low accuracy reads can therefore produce a perfect consensus sequence, provided their errors are random and the read depth is sufficiently large. Conversely, high accuracy reads can create an imperfect consensus regardless of the read depth, if they contain systematic errors.

Consensus accuracy is usually the main concern for applications with high read depth, such as genome assembly. For other applications, particularly those with low read depths, read accuracy is important. For example, clinical metagenomics may rely on data from a very small number of non-human reads [1], and inaccurate reads could make it harder to identify and characterise pathogens.

ONT have released multiple pore types during their history, but the R9.4 pore (and its minor revision R9.4.1) has been available for longest: from October 2016 to the present. Its release also corresponds to approximately when command-line basecallers became available - before that users needed to basecall with ONT's cloudbased Metrichor service. This study aims to quantify the performance of various basecalling tools developed for ONT's R9.4 pore and to explore the impact of model training on basecalling accuracy. It may provide guidance to those wishing to get the most out of ONT sequencing signals, and in particular could help readers to decide whether recent progress warrants re-basecalling older signal data with a newer basecaller or custom-trained model.

In this study, we tested four basecalling programs developed by ONT - Albacore, Guppy, Scrappie and Flappie and ran all available versions compatible with R9.4 reads. Albacore is a general-purpose basecaller that runs on CPUs. Guppy is similar to Albacore but can use GPUs for improved basecalling speed. While the two basecallers have coexisted for about a year, ONT has discontinued development on Albacore in favour of the more performant Guppy. Both Albacore and Guppy are only available to ONT customers via their community site (https:// community.nanoporetech.com). Scrappie (https://github. $\mathrm{com} /$ nanoporetech/scrappie) is an open-source basecaller which ONT describes as a 'technology demonstrator'. It has often been the first of ONT's basecallers to try new approaches, with successes later being incorporated into Albacore and Guppy. Scrappie is really two basecallers in one: Scrappie events, which carries out an event-segmentation step prior to basecalling with its neural network, and Scrappie raw, which basecalls directly from raw signal. We excluded some older versions of Scrappie events which rely on events first being defined by another program, as this requirement makes it not a standalone basecaller. Flappie (https://github.com/ nanoporetech/flappie) has recently replaced Scrappie and uses a CTC decoder to assign bases [2]. We also tested Chiron (https://github.com/haotianteng/Chiron), a thirdparty basecaller still under development that uses a deeper neural network than ONT's basecallers [3]. We excluded older basecallers no longer under development, such as Nanonet, DeepNano [4] and basecRAWller [5].

\section{Results and discussion \\ Default model performance}

Our primary benchmarking read set for assessing basecaller performance consisted of 15,154 whole genome sequencing reads from an isolate of Klebsiella pneumoniae (see 'Methods'). These reads ranged from $22-134 \mathrm{kbp}$ in length $(\mathrm{N} 50=37 \mathrm{kbp})$ and were $\sim 550 \mathrm{Mbp}$ in total size, equating to $100 \times$ read depth over the $5.5 \mathrm{Mbp}$ K. pneumoniae chromosome.

Albacore's history contained two major developments which resulted in distinct improvements in both read and consensus accuracy: in April 2017 (v1.0.1) and August 2017 (v2.0.2) (Fig. 1). The first was the addition of a transducer to the basecaller [6], which allowed for better homopolymer calls (see error profile details below). The second was the switch to raw basecalling, where sequence is called directly from raw signal without an event-segmentation step. After August 2017, Albacore's performance remained fairly constant with subsequent releases, achieving read accuracy of Q9.2 and consensus accuracy of Q21.9 with its final version (v2.3.4).

Guppy was publicly released in late 2017 (v0.3.0), and its accuracy stayed relatively constant and similar to that of Albacore for most of its version history (up to v1.8.5 in October 2018). The last tested version of Guppy (v2.2.3, released January 2019) performed worse on read accuracy (Q8.9) but better on consensus accuracy (Q22.8) using its default model. This version also comes with an optional 'flip-flop' model which has similar consensus accuracy 


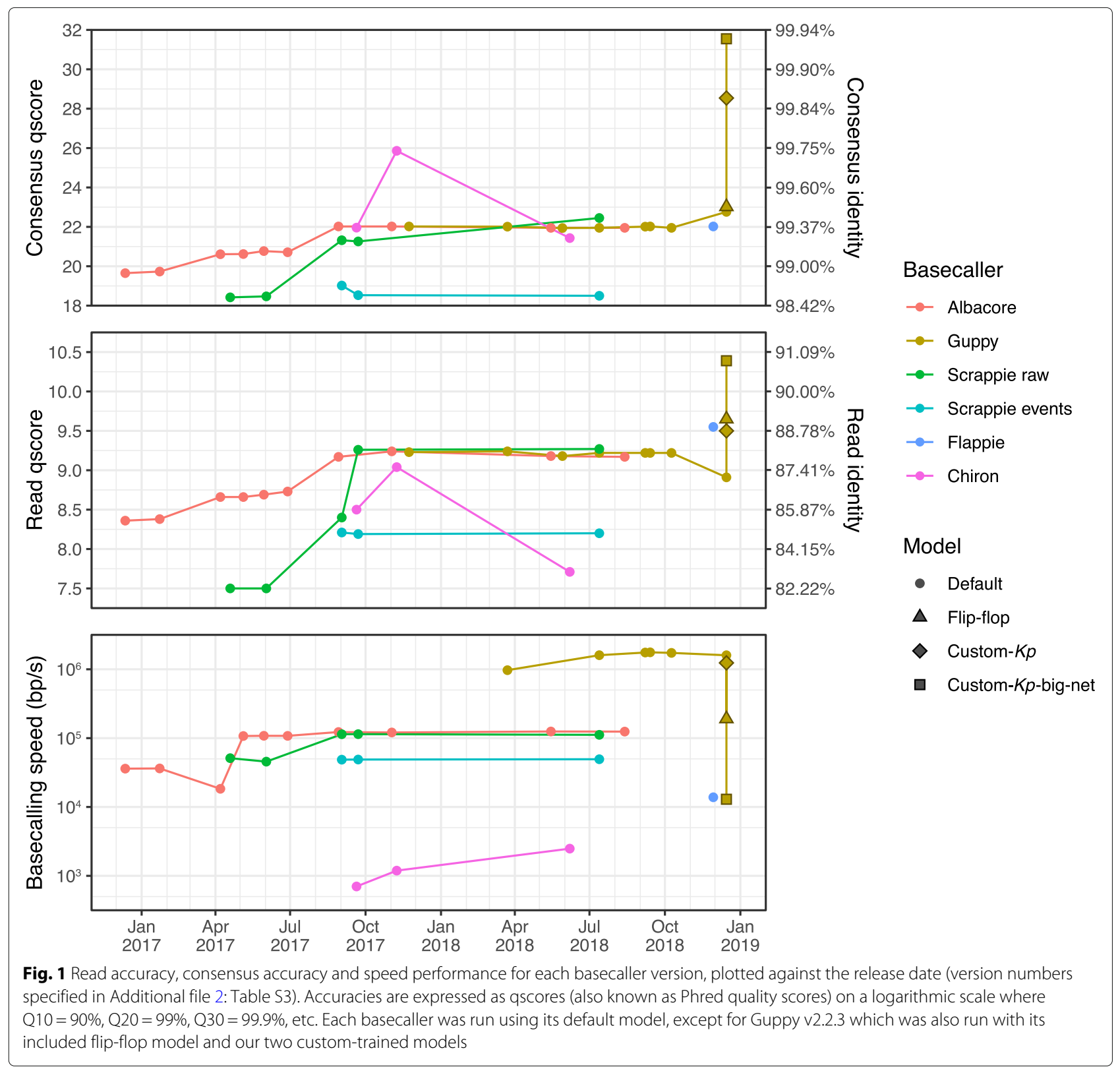

(Q23.0) but much better read accuracy (Q9.7). However, the flip-flop model's accuracy comes at the cost of speed performance - it is considerably slower than the default Guppy model, likely due to its larger size and complexity (Additional file 1: Figure S1).

Scrappie events was the worst-performing basecaller tested, likely due to its use of an outmoded eventsegmentation pre-processing step. Scrappie raw performed better, and the latest version (v1.4.1) performs similarly to Albacore (Q9.3 read accuracy and Q22.4 consensus accuracy). Scrappie's successor Flappie (released November 2018) showed an improvement in read accuracy (Q9.6) but not consensus accuracy (Q22.0).
Relative to ONT's basecallers, Chiron performed poorly on read accuracy (Fig. 1). Chiron v0.3 had the highest consensus accuracy (Q25.9) of all tested basecallers using their default models, but the latest version (v0.4.2) did not perform as well on our benchmarking set (Q7.7 read accuracy and Q21.4 consensus accuracy). This variation in accuracy for different versions of Chiron is explained by the taxonomy of the training data used to produce their default models (E. coli vs human, see 'Consensus error profiles').

While Albacore and Guppy are similar in terms of accuracy metrics, Guppy is an order of magnitude faster $(\sim 1,500,000 \mathrm{bp} / \mathrm{s}$ vs $\sim 120,000 \mathrm{bp} / \mathrm{s})$ due to its use of GPU 
acceleration (Fig. 1). Despite also using GPU acceleration, Chiron was the slowest basecaller tested $(\sim 2500 \mathrm{bp} / \mathrm{s})$, with the INF032 test set taking more than 2.5 days to basecall. This means that Chiron would take over a month to basecall a typical MinION yield of $10 \mathrm{Gbp}$, making it impractical for anything but very small read sets. Flappie also suffered from low speed performance $(\sim 14,000 \mathrm{bp} / \mathrm{s})$.

\section{Custom model performance}

Running Guppy v2.2.3 with our custom- $K p$ model (trained on unamplified DNA from $30 \mathrm{~K}$. pneumoniae, 10 other Enterobacteriaceae and 10 other Proteobacteria, see 'Methods' and Additional file 1: Figure S2) produced a modest increase in read accuracy (Q9.5) and a large increase in consensus accuracy $(\mathrm{Q} 28.5)$ for the benchmarking set, relative to the default model (Fig. 1). This demonstrates that there is a benefit for using taxonspecific training data. The default and custom- $K p$ models performed similarly in terms of speed.

Our custom-Kp-big-net model (trained on the same data as custom- $K p$ but with a larger neural network, Additional file 1: Figure S2) delivered even further improvements in both read accuracy (Q10.4) and consensus accuracy (Q31.6), showing that more complex neural networks also have the potential to give improved results, but at a cost to speed performance. The custom- $K p$-big-net model could not be run on the GPU because it uses neural network layers that are not pre-compiled into the Guppy program. It had to be run on the CPU instead, which along with the increased complexity of its neural network, resulted in a speed of $\sim 13,000 \mathrm{bp} / \mathrm{s}$ - two orders of magnitude slower than Guppy run with the default or custom- $K p$ models on the GPU.

Our custom-trained models were designed for K. pneumoniae and performed well on the K. pneumoniae benchmarking set. To see if these results generalise to other genomes (both K. pneumoniae and more distantly-related species), we also ran all available Guppy models on additional read sets (Fig. 2). The flip-flop model performed better than the default model for all genomes, with a mean improvement of +0.71 in the read qscore and +0.36 in the consensus qscore. The custom- $K p$ model performed much better than the default model for genomes in Enterobacteriaceae (K. pneumoniae and S. sonnei), with mean qscore improvements of +0.63 (read) and +4.72 (consensus). However, these benefits were not seen for species outside Enterobacteriaceae, where the mean qscore changes were 0.00 (read) and -1.64 (consensus). This taxon-specific improvement is likely due to the custom- $K p$ model's ability to more accurately call Dcmmethylation motifs which are found in Enterobacteriaceae [7] (see details below). The improved performance of custom- $K p$-big-net over the custom- $K p$ model was not taxon-dependent and showed mean qscore improvements of +1.01 (read) and +3.15 (consensus) across all genomes. In almost all cases, the custom- $K p$-big-net model produced the most accurate reads and consensus, the exception being $S$. aureus where the flip-flop model produced the most accurate reads.

Neither the custom-Kp model nor the custom- $K p$ big-net model use the new neural network architecture present in Guppy's flip-flop model. Presumably, a model based on this flip-flop architecture and trained on our custom training data would enjoy both the benefits of the flip-flop model (improved accuracy for all genomes) and of the custom- $K p$ model (improved accuracy for Enterobacteriaceae). However, the current version of Sloika (v2.1.0) does not allow for custom training with the flipflop architecture.

\section{Consensus error profiles}

In order to understand the impact of the various basecallers on different kinds of consensus basecalling errors, we quantified error profiles for the K. pneumoniae benchmarking genome in terms of the number of errors in Dcm-methylation sites, homopolymers and other sites (Fig. 3). All ONT basecallers performed poorly with Dcmmethylation sites when using the default models, and these made up a large proportion of consensus errors: for current versions of ONT basecallers $\sim 70 \%$ of the errors were in Dcm motifs and they created $\sim 0.4 \%$ error relative to the reference. This implies that the models were trained on data lacking Dcm methylation and have therefore not learned to call the sites reliably. Conversely, running Guppy v2.2.3 with our custom-trained models resulted in almost no Dcm errors $(\sim 0.002 \%)$ because Dcm methylation was well represented in our training set. The model included in versions 0.2 and 0.3 of Chiron was trained on E. coli reads [3] where Dcm modifications are expected [7], and those versions accordingly yielded very few Dcm errors $(<0.025 \%)$. The model in Chiron v0.4.2 was trained on human reads and thus yielded $0.29 \%$ Dcm errors (and more errors in general).

After Dcm motifs, incorrect homopolymer lengths made up the majority of errors (Fig. 3). ONT's progress on this front is evident in the performance of Albacore, for which consensus accuracy improvements over time have mostly come from a reduction in homopolymer errors, from $0.53 \%$ in v0.8.4 down to $0.13 \%$ in v2.3.4. More recently, Guppy v2.2.3 has shown further improvement, bringing homopolymer errors down to $0.07 \%$. While our custom- $K p$ model performed slightly worse than Guppy's default model for homopolymers $(0.10 \%)$, the custom- $K p$ big-net model performed better $(0.05 \%)$.

\section{Nanopolish performance}

Nanopolish can use the raw signal data to fix errors in a consensus sequence, and it includes special logic for 


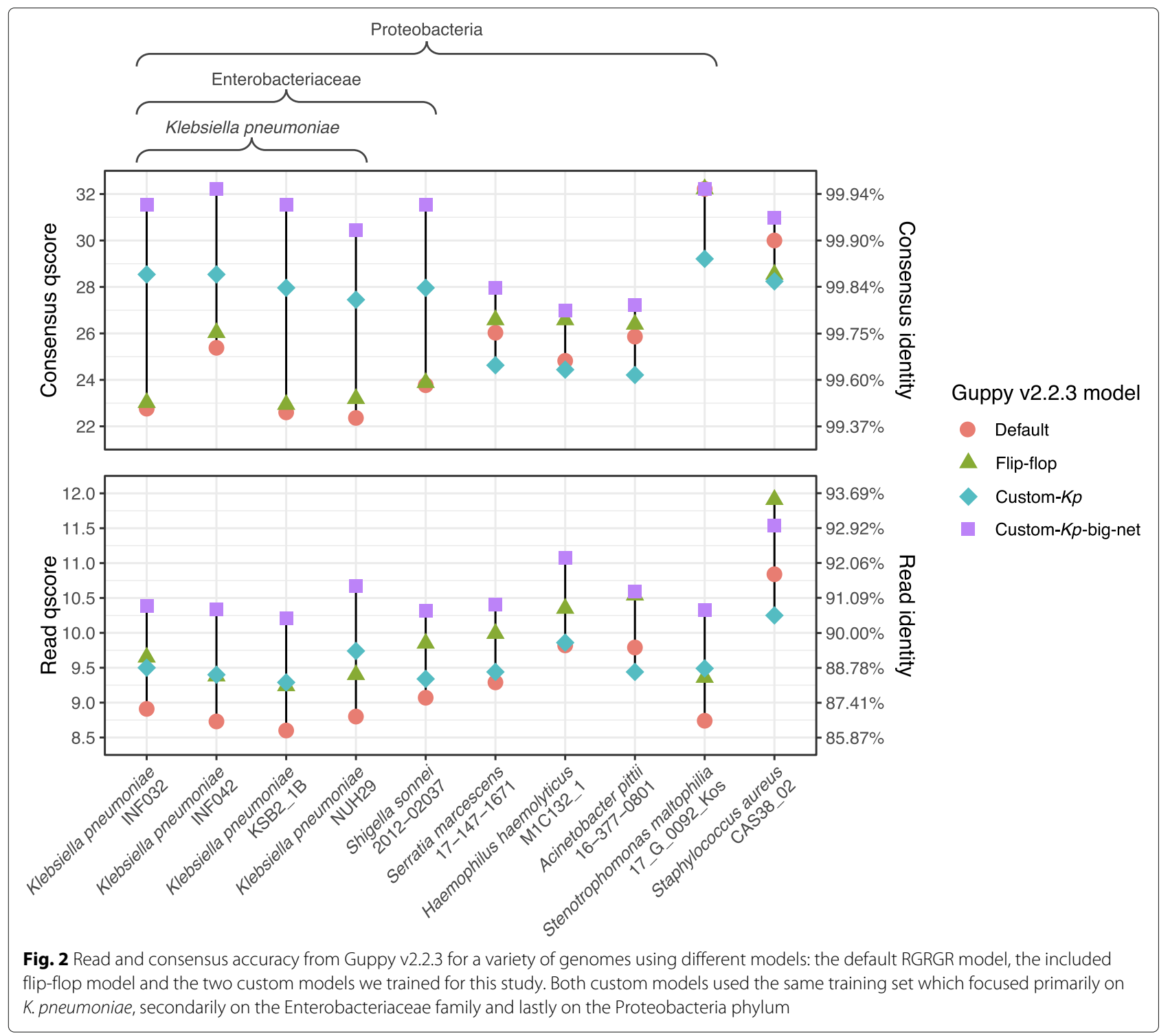

both Dcm methylation and homopolymers. We found that Nanopolish improved the consensus accuracy for our benchmarking set in nearly all cases, with the exception of our custom-Kp-big-net model (Fig. 4). The preNanopolish consensus accuracy was correlated with the post-Nanopolish accuracy $\left(R^{2}=0.580\right)$, indicating that a basecaller's consensus accuracy still matters even if Nanopolish is also used. While Nanopolish was able to account for Dcm methylation (using its - -methylation-aware option), it often only corrected $\sim 70-80 \%$ of Dcm errors (Additional file 1: Figure S3). Accordingly, the rate of Dcm errors in the pre-Nanopolish assembly was the strongest predictor of post-Nanopolish accuracy $\left(R^{2}=0.809\right)$, with the best results ( $>$ Q29 consensus) coming from the four basecallers with very low Dcm error rates (Chiron v0.2-v0.3 and both custom models). The effect of additional rounds of Nanopolish was tested on the Guppy v2.2.3 assembly and gave only a small increase in accuracy (from Q27.5 after one round to Q28.3 after four rounds, Additional file 1: Figure S4).

DNA base substitutions (often referred to as single nucleotide polymorphisms or SNPs) identified from pathogen whole genome sequence comparisons are now used routinely by public health and infection control laboratories to investigate suspected outbreaks of foodborne and other infectious diseases [8]. ONT platforms could potentially be useful in such investigations due to their portability and cost effectiveness for small sample sizes. However, the smallest number of substitution errors we encountered in a genome assembly (using Guppy v2.2.3 with the custom-Kp-big-net model and Nanopolish) was 


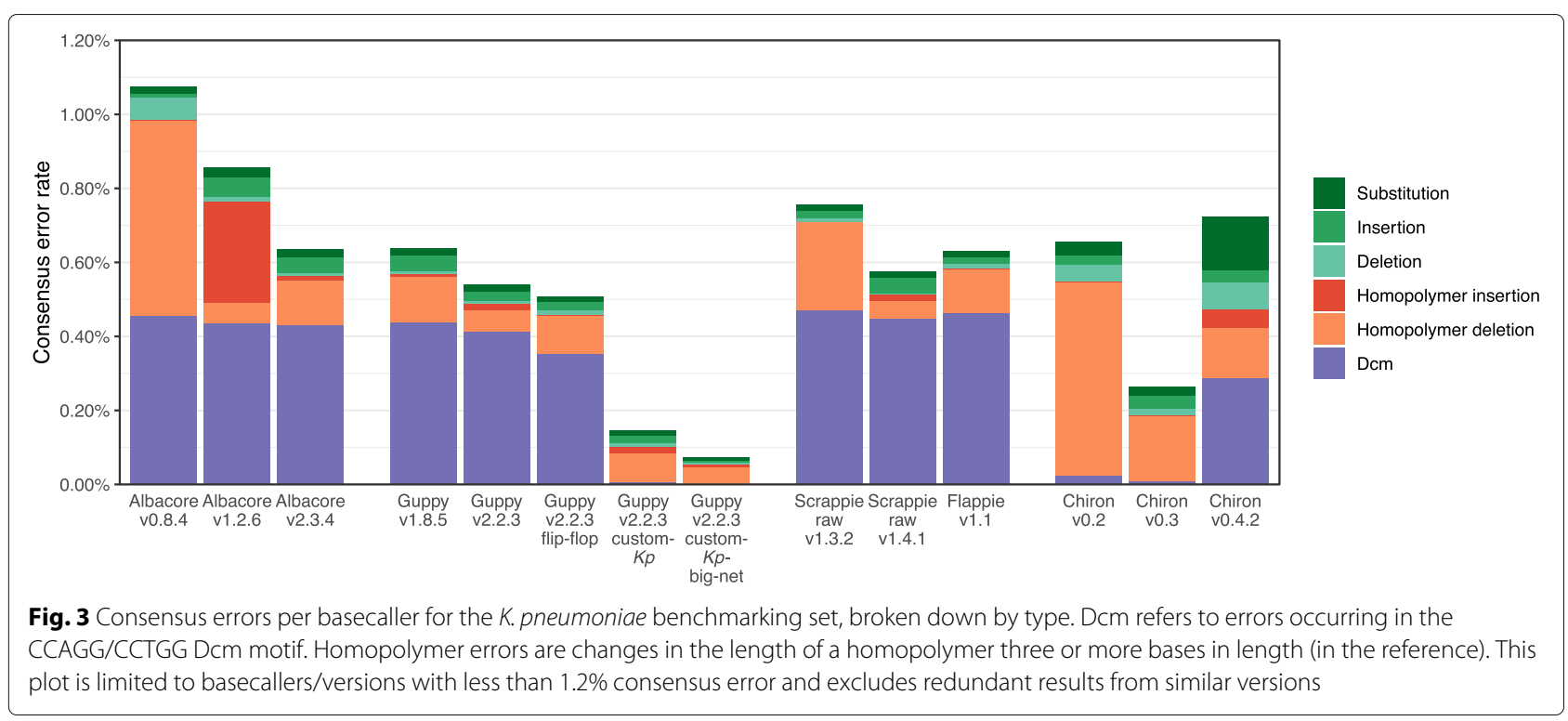

337 substitutions (Additional file 1: Figure S5, Additional file 2: Table S3), which at $\sim 10$ times the number of true SNPs expected between bacterial pathogen genomes linked to the same outbreak [8] would constitute an unacceptably high false positive rate for this application. While it is possible that tailored strategies for SNP calling from ONT reads could reduce the number of false positives, assembly-based sequence comparisons (which are frequently used in public health labs [9, 10]) would require dramatic improvements in basecalling accuracy.

\section{Conclusions}

Best results, in terms of both read and consensus accuracy for the K. pneumoniae benchmarking set, were obtained using Guppy v2.2.3 with a custom model trained on data mostly from the same species (Fig. 1). This superior performance seems to largely come from correct handling of Dcm methylation (Fig. 3). Since DNA modification patterns can differ between taxa, we propose our results may represent a more general trend: native DNA basecalling accuracy is best when the model was trained on native DNA from the same species or a sufficiently close relative to have similar DNA modifications. For example, a model trained on native human DNA may also perform well on native mouse DNA and vice versa, as CpG methylation is common to both species [11]. The benefits of custom training may further extend to direct RNA sequencing, where base modifications can be more extensive than in DNA $[12,13]$.

For most basecallers, full information is not disclosed on the taxa and DNA type (native or amplified) used to

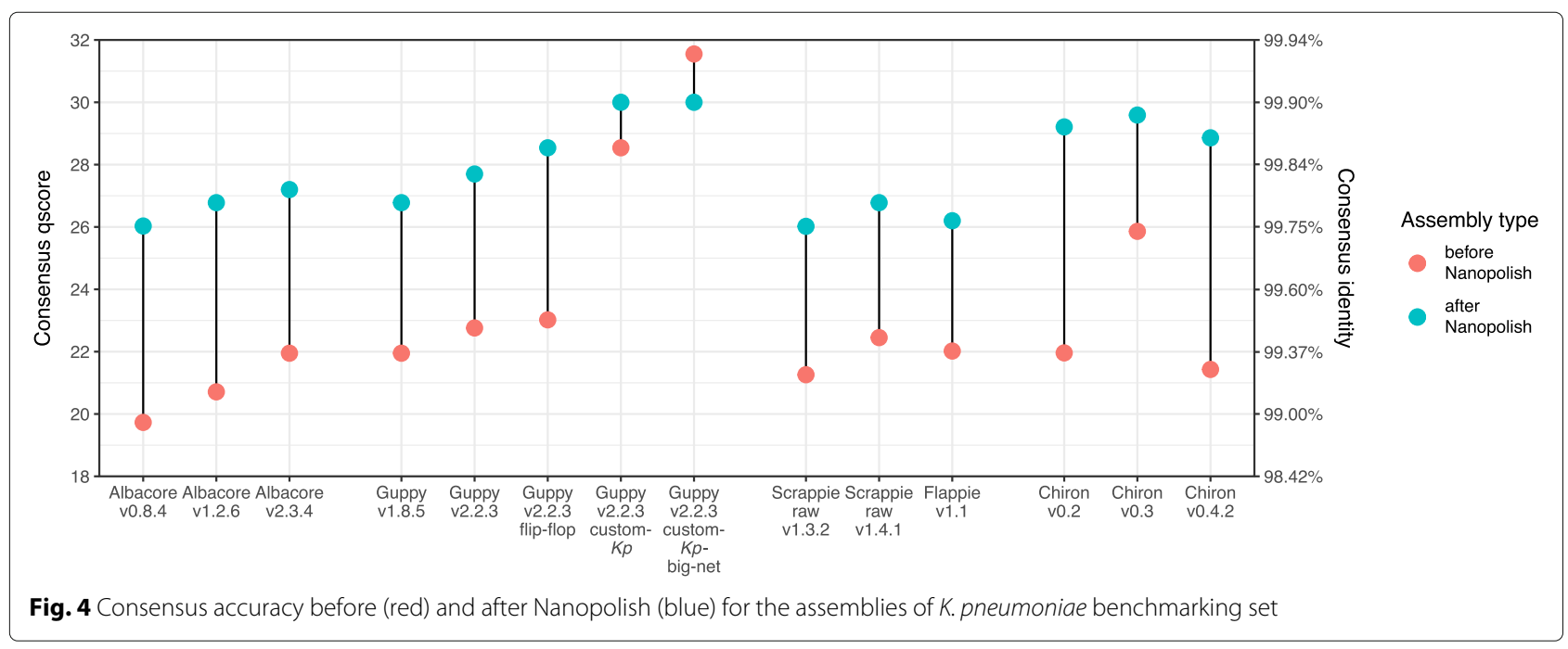


train the default model. We encourage developers to be more transparent in this regard and to consider providing multiple trained models when possible (e.g. amplified, native human, native $E$. coli) so users can choose one which most closely matches their organism. For users with sufficient quantities of training data, high-performance computers with GPUs and computational expertise, we recommend custom model training to maximise basecalling accuracy. Our custom-Kp-big-net model shows that even more accurate results are possible with bigger neural networks, including substantial improvements in read-level accuracy, but at a cost to speed performance (Fig. 1).

ONT sequencing has seen enormous gains in both yield and accuracy over the past few years, but our results show there is still much room for improvement. Across all basecallers, models and genomes, the best consensus accuracy we observed was Q32.2 (99.94\% identity). This equates, on average, to $\sim 3000$ errors in a $5 \mathrm{Mbp}$ genome. Many of these errors are substitutions which could lead to false-positive SNP calls, a potentially major impediment to outbreak investigations. In order to achieve a perfect bacterial genome assembly, the consensus accuracy will need to be orders of magnitude higher, e.g. Q70 (one error per $10 \mathrm{Mbp}$ ). Progress will likely come from many fronts: changes in technology and chemistry, improvements in basecalling, and development of post-assembly polishing tools. Until this goal is reached, hybrid assembly or polishing with Illumina reads will remain a necessity for researchers that depend on highly accurate sequences.

\section{Methods}

\section{Custom model training}

Sloika (https://github.com/nanoporetech/sloika) is ONT's neural network training toolkit which can be used to make models for use in Guppy. To explore the effect of the training set on basecalling performance, we used Sloika v2.1 to train a model ('custom-Kp') tailored to $K$. pneumoniae. The training reads came from 50 different isolate genomes: $30 \mathrm{~K}$. pneumoniae (chosen based on their phylogenetic uniqueness, each from a different lineage), 10 from other species of Enterobacteriaceae and 10 from other families of Proteobacteria (Additional file 1: Figure S2, Additional file 2: Table S6). Our training reads came from 20 different MinION runs, 10 of which were barcoded runs that contributed multiple genomes to the training set. Illumina reads were also available for all genomes, and we used SKESA [14] (v2.3.0) to produce high-quality contigs for each.

From an initial collection of 5,629,714 reads, we trimmed each read's signal at the fast 5 level, removing low-variance open-pore signal [5] and then an additional 2000 signal values from the start and end of the reads which served to remove adapter and barcode signals.
We also discarded short reads $(<50,000$ signal values), leaving 1,985,997 trimmed reads. We basecalled these reads (using Albacore v2.3.4) to select reads based on length ( $>5 \mathrm{kbp}$ ), completeness of alignment to the SKESA contigs ( $<30 \mathrm{bp}$ unaligned) and quality over a sliding window (no indel regions exceeding $25 \mathrm{bp}$ in size). This further reduced our set to 766,551 reads. We filtered the reads once more (following Sloika's training instructions: https://github.com/nanoporetech/sloika/blob/master/ scripts/example_training.sh), this time on the quality of the raw-signal-to-reference alignment. We set aside 20 of the resulting reads from each genome (1000 in total) to use as a validation set, leaving 226,166 for training the neural network. Sloika subdivides these reads into 'chunks' of 4000 signal values, of which there were $7,693,885$.

Using Sloika with this entire training set would have required hundreds of gigabytes of RAM, so we produced a fork of Sloika (https://github.com/rrwick/sloika) modified to load a random subset of the training data at periodic intervals. By only training on $5 \%$ of the data at a time and reloading a new $5 \%$ every 250 training batches, we were able to use the entirety of our training data while keeping RAM usage under $10 \mathrm{~GB}$. We trained the custom- $K p$ model for 47,500 batches (100 chunks per batch) on an NVIDIA P100 GPU, which took $36.5 \mathrm{~h}$.

Albacore, Guppy and Scrappie all use an architecture that ONT calls RGRGR - named after its alternating reverse-GRU and GRU layers (Additional file 1: Figure S1, top-left). To test whether more complex networks perform better, we modified ONT's RGRGR network by widening the convolutional layer and doubling the hidden layer size (Additional file 1: Figure S1, top-right). We trained this 'custom- $K p$-big-net' model on the same bacterial training set using our fork of Sloika. Training on an NVIDIA P100 GPU for 44,000 batches took 48 hours.

\section{Read sets}

To test basecaller performance, we used a set of reads generated using a MinION R9.4 flowcell to sequence native DNA extracted from the bacterium Klebsiella pneumoniae. The bacterial sample (isolate INF032, BioSample accession https://www.ncbi.nlm.nih.gov/biosample/ SAMEA3356991) was isolated from a urinary tract infection in an Australian hospital [15]. It was sequenced as part of a barcoded MinION run with other Klebsiella isolates, following the DNA extraction and library preparation protocol described in Wick et al. 2017 [16]. This particular sample was chosen for benchmarking basecalling accuracy because it had a good yield of ONT reads (see below) and contained no plasmids, making for a simpler assembly. It is not in the same K. pneumoniae lineage as any of the genomes used to train our custom models (Additional file 1: Figure S2). Since the sequenced 
DNA was native, it contains base modifications, the most relevant of which is Dcm methylation (conversion of cytosine to 5-methylcytosine at particular motifs) which is common in some species of Enterobacteriaceae [7]. Highquality Illumina reads were available for this sample: DNA was extracted and sequenced as $125 \mathrm{bp}$ paired-end reads via Illumina HiSeq 2000 at the Sanger Institute, producing 5,455,870 reads (ENA accession https://www.ebi. ac.uk/ena/data/view/ERR1023765) with 133× read depth over the INF032 chromosome [15]. This allowed us to generate an accurate reference sequence via hybrid assembly using Unicycler [16] (v0.4.0) which produced a single $5,111,537$ bp contig with a GC-content of $57.6 \%$. This reference was used as the ground truth sequence when assessing read and consensus accuracy for each basecaller (see below).

A subset of ONT reads was extracted for benchmarking basecallers against the reference genome of INF032. The entire barcoded MinION run containing INF032 was demultiplexed using Deepbinner [17], using its - -require_both option for high-precision demultiplexing. This produced 70,494 reads $(\sim 1.1 \mathrm{Gbp})$ for the barcode corresponding to INF032. We further reduced this dataset by basecalling with Guppy v1.6.0 (the current version at the time of read selection), aligning the resulting reads (using minimap2 [18] v2.14) to the INF032 reference genome and selecting those with $a \geq 22 \mathrm{kbp}$ alignment to the reference. This served to exclude 'junk' reads, very low-quality reads, improperly demultiplexed reads (belonging to a different isolate) and short reads. The threshold of $\geq 22 \mathrm{kbp}$ was chosen because it reduced the dataset to approximately $100 \times$ mean read depth for the INF032 genome while maintaining even coverage (Additional file 1: Figure S6). The resulting set contained 15,154 reads with lengths ranging from $22-$ $134 \mathrm{kbp}(\mathrm{N} 50=37 \mathrm{kbp})$ and totalling $\sim 550 \mathrm{Mbp}$. These reads are significantly longer than the longest repeat in the INF032 genome (the $\sim 5.5 \mathrm{kbp}$ rRNA operon) so each can be reliably mapped to its correct location on the reference genome. The Guppy qscore distributions (Additional file 1: Figure S6) show that while the selection process removed the lowest quality reads, the resulting reads still span a wide quality range, with $458(\sim 3 \%)$ falling below ONT's 'fail' threshold of Q7. This read set (hereafter referred to as the 'benchmarking set') was used with all basecallers and versions.

In addition to the primary $K$. pneumoniae benchmarking set, we also prepared test read sets for nine additional genomes. These include three other K. pneumoniae genomes and one genome each from six different bacterial species: Shigella sonnei, Serratia marcescens, Haemophilus haemolyticus, Acinetobacter pittii, Stenotrophomonas maltophilia and Staphylococcus aureus. As well as covering a wider range of species, these read sets span a wider date range (Feb 2017-Aug 2018) and include both R9.4 and R9.4.1 flowcells (Additional file 2: Table S1). They were prepared in the same manner as the benchmarking set, but we adjusted the alignment length threshold for each set as appropriate for the genome and read depth ( $3 \mathrm{kbp}$ to $33 \mathrm{kbp}$, see Additional file 2: Table S1). These read sets (hereafter referred to as the 'additional sets') were used to more thoroughly assess the current version of Guppy (v2.2.3) using its default model, its included flip-flop model and our two custom models (custom- $K p$ and custom- $K p$-big-net).

Despite the research interest in human DNA, we chose to limit our read sets to bacterial samples because they allow for a more confident 'ground truth' reference sequence against which to calculate accuracy than do complex eukaryote genomes. Bacterial genomes are haploid and have relatively low repetitive content (due in part to $>85 \%$ of sequence being protein coding), facilitating complete assembly and accurate Illumina-read polishing against which to calculate ONT basecalling accuracy. A recent study on de novo assembly of human ONT reads [6] found a $0.04 \%$ error rate between an Illuminapolished assembly and reference sequence. This level of uncertainty in the reference would make a Q50 consensus appear to be Q34, so estimates of consensus sequence accuracy could only provide a lower bound on the true value. Additionally, the small sizes of bacterial genomes make computational tasks more tractable and facilitate higher read depths. For these reasons, bacterial and phage DNA have been the standard in ONT accuracy testing and basecaller development, with previous studies either exclusively using bacterial DNA [4] or limiting human DNA to read-level analyses (i.e. excluding consensus-level analyses) $[3,5]$.

\section{Read accuracy}

We ran all basecallers on the benchmarking read set, with each producing either a FASTQ or FASTA file suitable for downstream analysis. To allow a comparison of speed performance, all basecalling was carried out on the same computer: six core (12 thread) Intel Xeon W-2135 CPU, 32 GB RAM, NVIDIA GTX 1080 GPU and 1 TB NVMe SSD. Basecalling was carried out using all $12 \mathrm{CPU}$ threads, or if supported by the basecaller, on the GPU.

To assess read accuracy, we aligned each basecalled read set to the reference INF032 genome using minimap2 [18] (v2.12). Each read's identity was defined as the number of matching bases in its alignment divided by the total alignment length including insertions and deletions, a.k.a. the 'BLAST identity' (https://lh3.github.io/2018/11/25/ on-the-definition-of-sequence-identity). Our read identity values were therefore based only on the fraction of each read which aligned to the reference ('per-read aligned fraction'), though if less than half of a read aligned it was 
given an identity of $0 \%(<6 \%$ of reads for all basecallers, see Additional file 2: Table S3). While this approach could overestimate the identity of each read (which may consist of both aligned and unaligned regions), our read selection process (only using reads with a long alignment to the reference, see above) resulted in reads with few unaligned regions: for our benchmarking set, each basecaller had a median per-read aligned fraction of $>99.5 \%$ (Additional file 2: Table S3), with most of the unaligned regions due to sequencing adapters and barcode sequences. Basing read identity on aligned regions should therefore provide a valid assessment of overall read identity for our test sets (although note this same approach may not be appropriate for other read sets where quality control has not been performed).

Another possible concern is that some basecallers might only output a selective subset of the data, i.e. instead of producing basecalled sequence for all reads, they might only output the higher-quality reads, resulting in an inflated average identity. To guard against this possibility, any reads missing from the output were given an identity of $0 \%$. However, of all basecallers and versions, only Albacore v0.7.5 (the oldest version tested) failed to output all 15,154 reads in our benchmarking set (3\% missing reads, see Additional file 2: Table S3). The distribution of perread identities for each basecaller was broad, with most reads in the range of $70 \%$ to $95 \%$. We thus defined each basecaller's overall read accuracy as the median identity across all reads in the set (counting the small fraction that were not reported or not alignable along $>50 \%$ of the read length as $0 \%$ identity).

\section{Consensus accuracy}

We used Rebaler (https://github.com/rrwick/Rebaler) to generate a consensus sequence from each basecalled read set. Rebaler is a reference-based assembler written for the purpose of comparing basecallers. It works by first replacing all parts of the reference genome using read sequences and then polishing the genome with multiple rounds of Racon [19]. This approach ensures that the assembled genome will have the same large-scale structure as the reference, but small-scale details (e.g. basecalls) will not be affected by the reference sequence. Even after multiple rounds, Racon does not always converge to the best possible sequence, so we used Rebaler with an iterative approach: running the assembly multiple times, each with shuffled input reads and a rotated (shifted start position) reference genome. We used 10 iterations, each resulting in a slightly different assembly which were then used as the 'reads' for a final Rebaler assembly. This iterative approach was able to reduce assembly errors by about $20 \%$ : individual Rebaler assemblies of the Guppy v2.2.3 reads had a consensus accuracy of 99.33\% (Q21.74) but the final iterative assembly had an accuracy of $99.47 \%$ (Q22.76).
To assess consensus accuracy, we divided each final Rebaler assembly into $10 \mathrm{kbp}$ pieces and analysed them in the same manner used for the reads: aligning to the reference and calculating identity. Each basecaller's overall consensus accuracy was defined as the median identity of these $10 \mathrm{kbp}$ pieces. The distributions of identities for assembly pieces were narrow and had standard deviations of $<0.1 \%$.

To classify consensus sequence errors by type, we aligned each assembly to the reference using NUCmer [20] (v3.1) and then classified each error based on the reference context. An error was classified as 'Dcm' if it occurred in a Dcm-methylation motif (CCAGG or CCTGG). It was classified as 'homopolymer insertion' or 'homopolymer deletion' if the error added or removed a base from a homopolymer three or more bases in length. If the previous categories did not apply, the error was classified as 'insertion, 'deletion' or 'substitution' as appropriate.

\section{Polishing}

This study is focused on the performance of basecalling tools, and post-assembly polishing with raw signal data is a separate topic that falls outside our scope. However, many users who produce ONT-only assemblies will run Nanopolish on their result, which uses the raw read signals to improve the consensus accuracy of an assembly. This raises the question: does basecaller choice matter if Nanopolish is used downstream? To assess this, we ran Nanopolish v0.10.2 [21] on each final Rebaler assembly and assessed the consensus accuracy as described above.

\section{Additional files}

Additional file 1: Supplementary figures. This file contains supplementary figures S1-S6. (PDF 3513 MB)

Additional file 2: Supplementary tables. This spreadsheet contains tables with information on each test read set, basecaller commands, all accuracy and error data results, and information on the training sets used for the custom models. (XLSX $85 \mathrm{~kb}$ )

Additional file 3: Review history. This document contains the peer review history for the manuscript. (DOCX $26 \mathrm{~kb}$ )

\section{Acknowledgements}

We would like to thank ONT staff for answering questions about commands and resource requirements to run the Sloika software to train custom models and the commands needed to deploy these models in Guppy.

Review history

The review history is available as Additional file 3.

\section{Authors' contributions}

RRW conducted the basecalling and performed the analyses. LMJ performed the sequencing and assisted with the data management. KEH was involved with the study's conception and design. All authors read and approved the final manuscript. 


\section{Funding}

This work was supported by the Bill \& Melinda Gates Foundation, Seattle and an Australian Government Research Training Program Scholarship. KEH is supported by a Senior Medical Research Fellowship from the Viertel Foundation of Victoria.

\section{Availability of data and materials}

All read sets (raw fast 5 and basecalled), assemblies, reference genomes, training data and custom models used in this paper are available via figshare (https://doi.org/10.26180/5c5a5f5ff20ed, https://doi.org/10.26180/ 5c5a5fa08bbee, https://doi.org/10.26180/5c5a5fb6ac10f, https://doi.org/10. 26180/5c5a5fc61e7fa, https://doi.org/10.26180/5c5a5fcf72e40, https://doi. org/10.26180/5c5a7292227de) [22-27].

Scripts used to perform basecalling and analysis are available via GitHub: https://github.com/rrwick/Basecalling-comparison [28].

\section{Ethics approval and consent to participate}

Not applicable.

\section{Consent for publication}

Not applicable.

\section{Competing interests}

In July 2018, Ryan Wick attended a hackathon in Bermuda at ONT's expense. ONT also paid his travel, accommodation and registration to attend the London Calling (2017) and Nanopore Community Meeting (2017) events as an invited speaker.

\section{Author details}

${ }^{1}$ Department of Infectious Diseases, Central Clinical School, Monash University, Melbourne 3004, Australia. ${ }^{2}$ London School of Hygiene \& Tropical Medicine, London WC1E 7HT, UK.

\section{Received: 5 March 2019 Accepted: 30 May 2019}

Published online: 24 June 2019

\section{References}

1. Charalampous T, Richardson H, Kay GL, Baldan R, Jeanes C, Rae D, Grundy S, Turner DJ, Wain J, Leggett RM, Livermore DM, O'Grady J. Rapid diagnosis of lower respiratory infection using Nanopore-based clinical metagenomics. bioRxiv. 2018:387548. https://doi.org/10.1101/387548

2. Graves A, Fernández S, Gomez F, Schmidhuber J. Connectionist temporal classification: Labelling unsegmented sequence data with recurrent neural networks. In: ICML '06 Proceedings of the $23 \mathrm{rd}$ International Conference on Machine Learning; 2006. p. 369-76. https:// doi.org/10.1145/1143844.1143891. http://arxiv.org/abs/1607.03597.

3. Teng H, Cao MD, Hall MB, Duarte T, Wang S, Coin LJM. Chiron: Translating nanopore raw signal directly into nucleotide sequence using deep learning. GigaScience. 2018;7(5):1-9. https://doi.org/10.1093/ gigascience/giy037.

4. Boža V, Brejová B, Vinař T. DeepNano: Deep recurrent neural networks for base calling in MinION Nanopore reads. PLOS ONE. 2017;12(6):1-13. https://doi.org/10.1371/journal.pone.0178751.

5. Stoiber M, Brown J. BasecRAWller: Streaming nanopore basecalling directly from raw signal. bioRxiv. 20171-15. https://doi.org/10.1101/ 133058.

6. Jain M, Koren S, Quick J, Rand AC, Sasani TA, Tyson JR, Beggs AD, Dilthey AT, Fiddes IT, Malla S, Marriott H, Miga KH, Nieto T, O'Grady J, Olsen HE, Pedersen BS, Rhie A, Richardson H, Quinlan A, Snutch TP, Tee L, Paten B, Phillippy AM, Simpson JT, Loman NJ, Loose M. Nanopore sequencing and assembly of a human genome with ultra-long reads. Nat Biotechnol. 2018;36:338-45. https://doi.org/10.1038/nbt.4060.

7. Smith JW, Gomez-Eichelmann MC, Levy-Mustri A, Ramirez-Santos J. Presence of 5-methylcytosine in CC(A/T)GG sequences (Dcm methylation) in DNAs from different bacteria. J Bacteriol. 1991;173(23): 7692-4. https://doi.org/10.1093/aesa/91.3.239.

8. Pightling AW, Pettengill JB, Luo Y, Baugher JD, Rand H, Strain E. Interpreting whole-genome sequence analyses of foodborne bacteria for regulatory applications and outbreak investigations. Front Microbiol. 2018;9:1-13. https://doi.org/10.3389/fmicb.2018.01482.
9. Yoshida CE, Kruczkiewicz P, Laing CR, Lingohr EJ, Victor P. The Salmonella in silico typing resource (SISTR): An open web-accessible tool for rapidly typing and subtyping draft Salmonella genome assemblies. PLOS ONE. 2016;11(1):0147101. https://doi.org/10.1371/journal.pone.0147101.

10. Schjørring S, Gillesberg Lassen S, Jensen T, Moura A, Kjeldgaard JS, Müller L, Thielke S, Leclercq A, Maury MM, Tourdjman M, Donguy MP, Lecuit M, Ethelberg S, Nielsen EM. Cross-border outbreak of listeriosis caused by cold-smoked salmon, revealed by integrated surveillance and whole genome sequencing (WGS), Denmark and France, 2015 to 2017. Eurosurveillance. 2017;22(50):1-5. https://doi.org/10.2807/1560-7917.ES. 2017.22.50.17-00762.

11. Chinwalla AT, Cook LL, Delehaunty KD, Fewell GA, Fulton LA, et al. Initial sequencing and comparative analysis of the mouse genome. Nature. 2002;420(6915):520-62. https://doi.org/10.1038/nature01262.

12. Garalde DR, Snell EA, Jachimowicz D, Sipos B, Lloyd JH, Bruce M, Pantic N, Admassu T, James P, Warland A, Jordan M, Ciccone J, Serra S, Keenan J, Martin S, McNeill L, Wallace EJ, Jayasinghe L, Wright C, Blasco J, Young S, Brocklebank D, Juul S, Clarke J, Heron AJ, Turner DJ. Highly parallel direct RNA sequencing on an array of nanopores. Nat Methods. 2018;15(3):201. https://doi.org/10.1038/nmeth.4577.

13. Gilbert WV, Bell TA, Schaening C. Messenger RNA modifications: Form, distribution, and function. Science. 2016;352(6292):1408-12. https://doi. org/10.1126/science.aad8711.

14. Souvorov A, Agarwala R, Lipman DJ. SKESA: Strategic k-mer extension for scrupulous assemblies. Genome Biol. 2018;19(1):153. https://doi.org/10. 1186/s13059-018-1540-z.

15. Gorrie CL, Mirceta M, Wick RR, Judd LM, Wyres KL, Thomson NR, Strugnell RA, Pratt NF, Garlick JS, Watson KM, Hunter PC, McGloughlin SA, Spelman DW, Jenney AWJ, Holt KE. Antimicrobial-resistant Klebsiella pneumoniae carriage and infection in specialized geriatric care wards linked to acquisition in the referring hospital. Clin Infect Dis. 2018;67(2): 161-70. https://doi.org/10.1093/cid/ciy027.

16. Wick RR, Judd LM, Gorrie CL, Holt KE. Completing bacterial genome assemblies with multiplex MinION sequencing. Microb Genom. 2017;3(10):1-7. https://doi.org/10.1099/mgen.0.000132.

17. Wick RR, Judd LM, Holt KE. Deepbinner: Demultiplexing barcoded Oxford Nanopore reads with deep convolutional neural networks. PLoS Comput Biol. 2018;14(11):1006583. https://doi.org/10.1371/journal.pcbi.1006583.

18. Li H. Minimap2: Pairwise alignment for nucleotide sequences. Bioinformatics. 2018;34(18):3094-100. https://doi.org/10.1093/ bioinformatics/bty191.

19. Vaser R, Sović I, Nagarajan N, Šikić M. Fast and accurate de novo genome assembly from long uncorrected reads. Genome Res. 2017;27(5): 737-46. https://doi.org/10.1101/gr.214270.116.

20. Kurtz S, Phillippy A, Delcher AL, Smoot M, Shumway M, Antonescu C, Salzberg SL. Versatile and open software for comparing large genomes. Genome Biol. 2004;5(2):12. https://doi.org/10.1186/gb-2004-5-2-r12.

21. Loman NJ, Quick J, Simpson JT. A complete bacterial genome assembled de novo using only nanopore sequencing data. Nat Methods. 2015;12(8):733-5. https://doi.org/10.1038/nmeth.3444.

22. Wick RR, Judd LM, Holt KE. Training data. Figshare. 2019. https://doi.org/ 10.26180/5c5a5f5ff20ed.

23. Wick RR, Judd LM, Holt KE. Trained models. Figshare. 2019. https://doi. org/10.26180/5c5a5fc61e7fa.

24. Wick RR, Judd LM, Holt KE. Raw fast5s. Figshare. 2019. https://doi.org/10. 26180/5c5a5fa08bbee.

25. Wick RR, Judd LM, Holt KE. Basecalled reads. Figshare. 2019. https://doi. org/10.26180/5c5a7292227de.

26. Wick RR, Judd LM, Holt KE. Assemblies. Figshare. 2019. https://doi.org/10. 26180/5c5a5fb6ac10f.

27. Wick RR, Judd LM, Holt KE. Reference genomes. Figshare. 2019. https:// doi.org/10.26180/5c5a5fcf72e40.

28. Wick RR, Judd LM, Holt KE. Analysis scripts. GitHub. 2019. https://doi.org/ 10.5281/zenodo.1188469.

\section{Publisher's Note}

Springer Nature remains neutral with regard to jurisdictional claims in published maps and institutional affiliations. 